\title{
Correction to: Solitary fibrous tumor: a case series identifying pathological adverse factors-implications for risk stratification and classification
}

Isidro Machado ${ }^{1}$ - Gema Nieto Morales ${ }^{2}$ - Julia Cruz ${ }^{1}$ - Javier Lavernia ${ }^{3}$. Francisco Giner ${ }^{4}$ - Samuel Navarro ${ }^{5}$. Antonio Ferrandez ${ }^{5}$. Antonio Llombart-Bosch ${ }^{5}$

Published online: 26 October 2019

(C) Springer-Verlag GmbH Germany, part of Springer Nature 2019

\section{Correction to: Virchows Archiv}

https://doi.org/10.1007/s00428-019-02660-3

The original version of this article, unfortunately, contained errors.

In Tables 4 and 5, there were entries that were not properly aligned on its designated rows. The correct tables are shown in this article. The original article has been corrected.

The online version of the original article can be found at https://doi.org/ 10.1007/s00428-019-02660-3

Isidro Machado

isidro.machado@uv.es

1 Pathology Department, Instituto Valenciano de Oncología, Valencia Spain

2 Molecular Biology, Pathology Department, University of Valencia, Valencia Spain

3 Department of Oncology, Instituto Valenciano de Oncología, Valencia Spain

4 Pathology Department, University Hospital "La Fe", Valencia Spain

5 Pathology Department, University of Valencia, Valencia Spain 
Table 4 Solitary fibrous tumor. Immunohistochemical data(N=28)

\begin{tabular}{|c|c|c|}
\hline Variables & $\mathrm{N}$ & $\%$ \\
\hline \multicolumn{3}{|l|}{ STAT6 } \\
\hline Positive & 28 & 100 \\
\hline Negative & 0 & 0 \\
\hline \multicolumn{3}{|l|}{$\mathrm{Ki}-67$} \\
\hline$\geq 10$ & 9 & 32.1 \\
\hline$<10$ & 19 & 67.9 \\
\hline \multicolumn{3}{|l|}{ CD99 } \\
\hline Positive & 16 & 57.1 \\
\hline Negative & 12 & 42.9 \\
\hline \multicolumn{3}{|l|}{ CD34 } \\
\hline Positive & 26 & 92.9 \\
\hline Negative & 2 & 7.1 \\
\hline \multicolumn{3}{|l|}{ Bcl2 } \\
\hline Positive & 19 & 67.9 \\
\hline Negative & 9 & 32.1 \\
\hline \multicolumn{3}{|l|}{ INSM2 } \\
\hline Positive & 6 & 21.4 \\
\hline Negative & 21 & 75 \\
\hline Non informative & 1 & 3.6 \\
\hline \multicolumn{3}{|c|}{ Synaptophysin, Chromogranin-A and Myogenin } \\
\hline Positive & 0 & 0 \\
\hline Negative & 28 & 100 \\
\hline \multicolumn{3}{|l|}{ Desmin } \\
\hline Positive & 4 & 14.3 \\
\hline Negative & 24 & 85.7 \\
\hline \multicolumn{3}{|l|}{ p16 } \\
\hline Positive & 5 & 17.9 \\
\hline Negative & 23 & 82.1 \\
\hline \multicolumn{3}{|l|}{ HTER } \\
\hline Positive & 13 & 46.4 \\
\hline Negative & 14 & 50 \\
\hline Non informative & 1 & 3.6 \\
\hline
\end{tabular}

Table 5 Solitary fibrous tumor. Risk stratification system of recurrence/ metastasis $(\mathrm{N}=28)$

\begin{tabular}{llr}
\hline Variables & $\mathrm{N}$ & $\%$ \\
\hline Demicco system (USA) & & \\
$\quad$ High risk & 6 & 21.4 \\
$\quad$ Intermediate risk & 8 & 28.6 \\
$\quad$ Low risk & 13 & 46.4 \\
$\quad$ Non informative & & 3.6 \\
Diebol system (Switzerland) & 6 & 21.4 \\
$\quad$ High risk & 22 & 78.6 \\
$\quad$ Low risk & & \\
Sales system (France) & 10 & 35.8 \\
$\quad$ High/unfavorable & 18 & 64.2 \\
$\quad$ Low/favorable & & 35.8 \\
Pasquali (UK and Italy/Milan) & 10 & 32.1 \\
$\quad$ High risk & 9 & 32.1 \\
$\quad$ Intermediate risk & 9 & \\
Low risk & &
\end{tabular}

Publisher's note Springer Nature remains neutral with regard to jurisdictional claims in published maps and institutional affiliations. 